\title{
A Compact and Stable Frequency Selective Surface for WLAN Applications
}

\author{
R. B. Moreira \\ State University of Southwest \\ Bahia (UESB) \\ DCT - CPDS \\ Jequié - BA, Brazil
}

\author{
A. F. dos Santos \\ Federal University of \\ Recôncavo da Bahia (UFRB) \\ CETENS \\ Feira de Santana - BA, Brazil
}

\author{
R. H. C. Maniçoba \\ State University of Southwest \\ Bahia (UESB) \\ DCT - CCSI - CPDS \\ Jequié - BA, Brazil
}

\begin{abstract}
In this paper, a single-layer bandstop Frequency Selective Surface (FSS) is proposed for Wireless Local Area Networks (WLAN) Applications. The unit cell of the proposed FSS consists of a modification in a square patch element by the insertion of triangular shaped slots. The designs demonstrate a wide $3.8 \mathrm{GHz}$ stopband in the WLAN frequency range. Moreover, it shows angular stability at various angles of incidence up to $40^{\circ}$.
\end{abstract}

\section{Keywords}

Frequency selective surfaces, angular stability, polarization independence, WLAN.

\section{INTRODUCTION}

Frequency Selective Surfaces (FSS) can be defined as a 2D planar periodic array structure of identical elements, mainly used for filtering purpose. These elements can be of aperture type, patch type or an array of all-dielectric elements. The FSS structure can exhibit bandstop or bandpass characteristics, which depends primarily on the type and the geometry of the structure in one period called unit cell [1], [2], [3], [4].

FSS have been investigated and studied by several researchers over the years for a wide variety of applications, particularly in microwave and communications applications. One of its main uses is the integration with antennas systems acting as radomes and subreflectors, the latter is applied to separate electromagnetic waves into different frequency bands. These applications also includes bandpass and bandstop spatial filters, absorbers, artificial electromagnetic bandgap materials, uses as superstrate of antenna or antenna array to enhance the directivity and gain [5], [6], [7]. Currently, FSS with polarization independent, angular stability, and multiband or wideband characteristics (transmission or reflection), has received special attention [8], [9], [10].

UWB systems require UWB FSS for broadband shielding due its short pulse transmission and high data rate [8].

In this paper, a single-layer bandstop FSS providing a wide bandwidth of $3.80 \mathrm{GHz}$ approximately, from $2.25 \mathrm{GHz}$ to $6.05 \mathrm{GHz}$ with resonance frequency at $5.25 \mathrm{GHz}$, for a $10 \mathrm{~dB}$ insertion loss reference level, is proposed. A structure of the element in the unit cell provides a good angular stability and similar transmission characteristics for both $\mathrm{X}$-polarized electric field (TE Mode) and for Y-polarized electric filed (TM Mode) for a normal incidence.

\section{FSS STRUCTURE}

The proposed structure is a simple modification of conventional square patch. The element geometry used as unit cell for the proposed FSS structure is shown in Figure 1, and the design of the FSS element starts with a traditional square patch geometry shown in Figure 2(a). Adding four triangular shaped slots to the square patch it will obtain a structure called intermediate element shown in Figure 2(b), and then adding more four triangular shaped slots to the intermediate element, it will obtain the proposed element for the FSS structure.

The process uses four isosceles triangles slots to obtain the intermediate element, and from this more four isosceles triangles slots, these new slots use as a measured of one side the average value for the first one base (the biggest side), and are connected to obtain this new element.

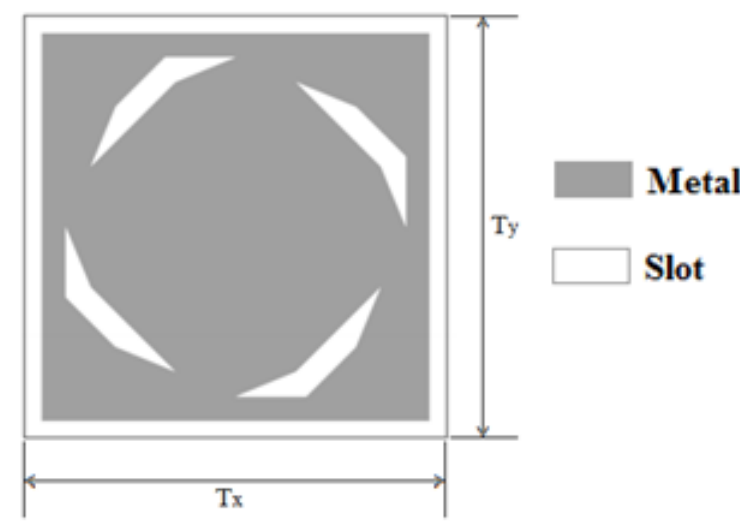

Fig 1: A unit cell of the proposed FSS structure.

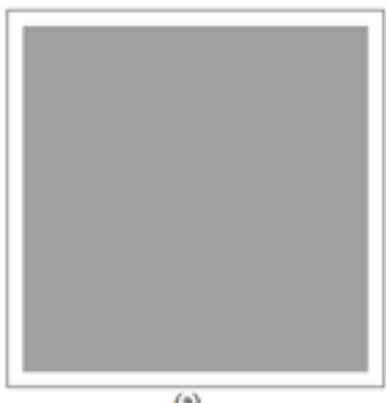

(a)

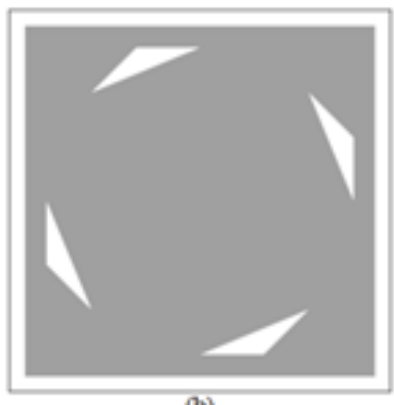

(b)

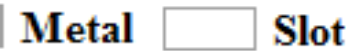

Fig 2: A unit cell of the: (a) square patch and (b) the intermediate element. 
As shown in Figure 1, the periodicity is $T x=T y=24.0 \mathrm{~mm}$ and this value was used as periodicity for all elements in Figure 2 too. A traditional square patch with side equals to $22.0 \mathrm{~mm}(22.0 \mathrm{~mm}$ X $22.0 \mathrm{~mm})$ can be seen in Figure 2(a). The FSS structure is mounted on a dielectric substrate with relative permittivity of 10.2 with dielectric loss tangent of 0.0035 and the thickness dielectric substrate is $1.28 \mathrm{~mm}$. All the dimensions of the triangular shaped slots can be seen in details in the Figure 3, for both intermediate and proposed elements.

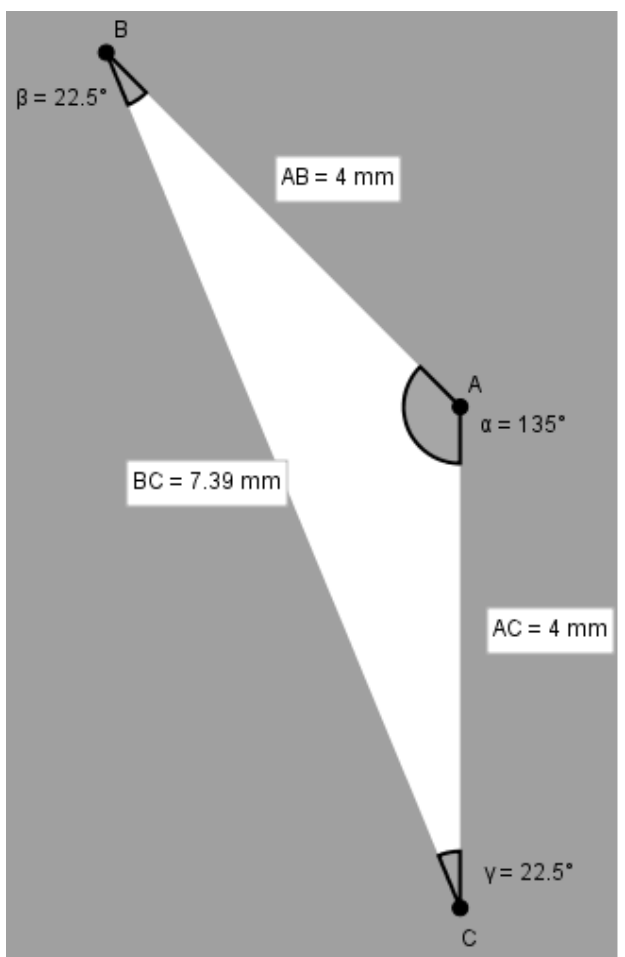

(a)

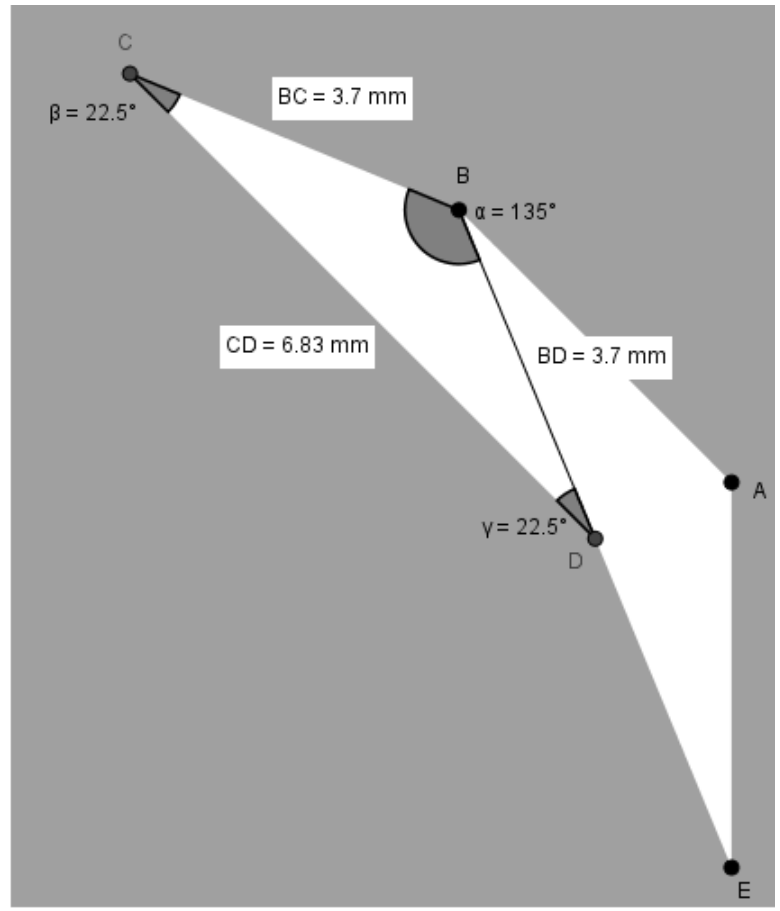

(b)

Fig 3: Dimensions of the slots: (a) intermediate element and (b) proposed FSS structure.

\section{RESULTS}

To investigate the performance of the proposed FSS in terms of transmission characteristics, the structure was simulated using Method of Moments (MoM), and the results are presented in Figures $4-6$. In Figure 4 can be observed a transmission characteristics for proposed FSS structure with resonance frequency at $5.25 \mathrm{GHz}(-53.28 \mathrm{~dB})$, it has a bandwidth equals to $3.80 \mathrm{GHz}$, from $2.25 \mathrm{GHz}$ to $6.05 \mathrm{GHz}$, for a $10 \mathrm{~dB}$ insertion loss reference level. In Figure 5 is shown the results, in terms of transmission coefficients, for both TE and TM modes. It is possible to observe identical results for both modes, that is, polarization independence.

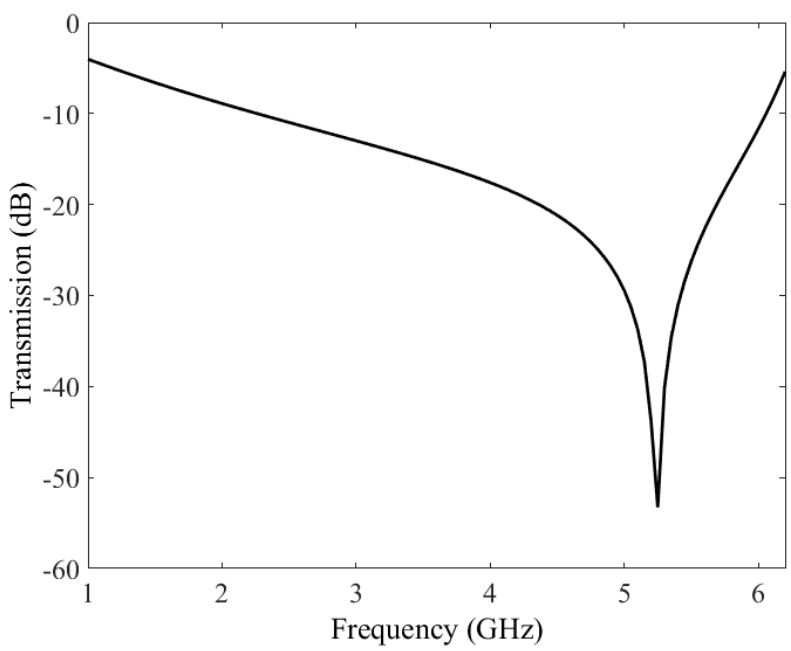

Fig 4: Obtained transmission coefficient for the proposed FSS.

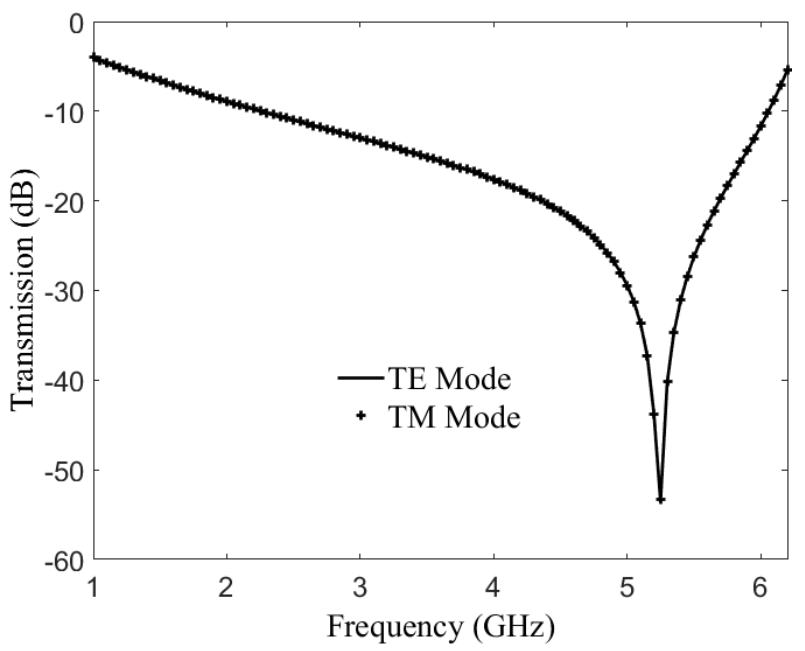

Fig 5: Transmission coefficients for TE mode and TM mode for the proposed FSS.

In Figure 6 is shown the transmission characteristics for the proposed FSS for different angles of incidence. In this case, it is possible to observe a good angular stability, for a $10 \mathrm{~dB}$ insertion loss reference level, from $0^{\circ}$ up to $40^{\circ}$.

The proposed FSS can be used in many WLAN applications, and it has very attractive characteristics, such as good angular stability. Several frequency channels used in WLAN are within the bandwidth obtained for the proposed FSS: $2.4 \mathrm{GHz}$ (IEEE $802.11 \mathrm{~b} / \mathrm{g} / \mathrm{n}$ ), $3.65 \mathrm{GHz}$ (IEEE 802.11y), $4.9 \mathrm{GHz}$ (IEEE $802.11 \mathrm{j}), 5 \mathrm{GHz}(802.11 \mathrm{a} / \mathrm{h} / \mathrm{j} / \mathrm{n} / \mathrm{ac})$ and $5.9 \mathrm{GHz}($ IEEE $802.11 \mathrm{p})$ 


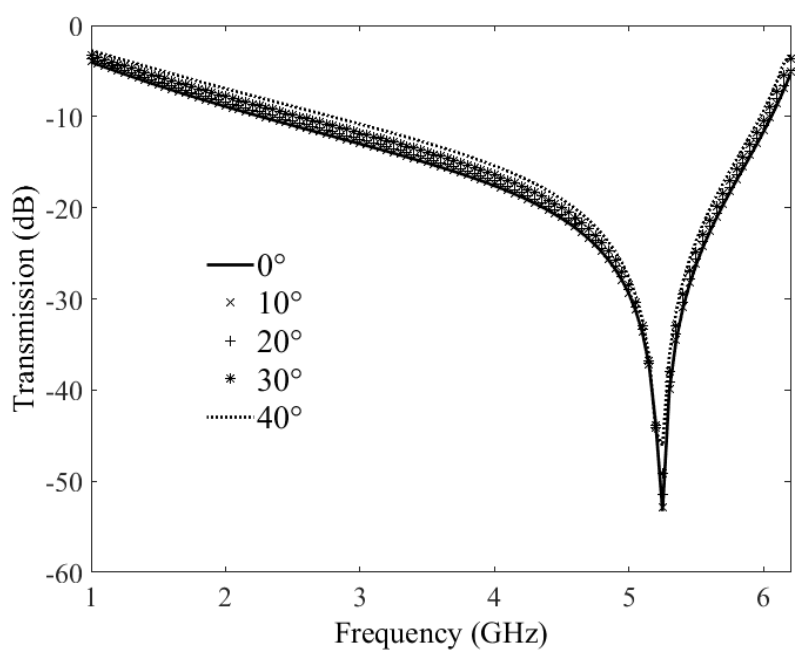

Fig 6: Transmission coefficients for different angles of incidence for the proposed FSS.

\section{CONCLUSION}

A compact single-layer bandstop FSS is proposed in this paper. A simple modification on a traditional square patch FSS was made, triangular shaped slots was inserted into it. This process makes it possible to obtain a structure for WLAN applications only. This FSS structure has the advantage of polarization independent for a normal incidence, angular stability and wideband with WLAN frequency applications. An investigation with the proposed structure in a multilayer format can be made to observe the behavior in terms of transmission and reflection coefficients and its bandwidths. It can also be investigated a new or different (with other dimensions) format for the slot used in the proposed structure.

\section{ACKNOWLEDGMENTS}

This work is supported by Foundation for Research Support of the State of Bahia (FAPESB - Fundação de Amparo à Pesquisa do Estado da Bahia).

\section{REFERENCES}

[1] T. K. Wu, Frequency Selective Surface and Grid Array, Wiley, New York, 1995.
[2] B. A. Munk, Frequency Selective Surface: Theory and Design, Wiley, New York, 2000.

[3] A. L. P. S. Campos, A. G. d'Assunção and L. M. Mendonça, "Scattering by FSS on anisotropic substrate for TE and TM excitation", IEEE Transactions on Microwave Theory and Techniques, 50 (1), $72-76$, 2002.

[4] J. H. Barton, C. R. Garcia, E. A. Berry, R. G. May, D. T. Gray and R. C. Rumpf, "All-Dielectric Frequency Selective Surface for High Power Microwaves", IEEE Transactions on Antennas and Propagation, 62 (7), 3652 $-3656,2014$.

[5] D. B. Brito, A. G. d'Assunção, R. H. C. Maniçoba and X. Begaud, "Metamaterial inspired Fabry-Pérot antenna with cascaded frequency selective surfaces", Microwave and Optical technology Letters, 55 (5), 981 - 985, 2013.

[6] H. Zhu, Y. Yu, X. Li and B. Ai, "A Wideband and High Gain Dual-Polarized Antenna Design by FrequencySelective Surface for WLAN Application", Progress In Electromagnetics Research C (PIER C), 54, 57 - 66, 2014.

[7] D. Gangwar, S. Das, R. L. Yadava and B. K. Kanaujia, "Circularly polarized inverted stacked high gain antenna with frequency selective surface", Microwave and Optical technology Letters, 58 (3), 732 - 740, 2016.

[8] R. Sivasamy, B. Moorthy, M. Kanagasabai, J. V. George, L. Lawrance and D. B. Rajendran, "Polarizationindependent single-layer ultra-wideband frequencyselective surface", International Journal of Microwave and Wireless Technologies, 9 (1), 93 - 97, 2017.

[9] I. Sohail, Y. Ranga, L. Matekovits, K. P. Esselle and S. G. Hay, "A Low-Profile Single-Layer UWB Polarization Stable FSS for Electromagnetic Shielding Applications", In Proceedings of the 2014 iWAT International Workshop on Antenna Technology, 220 - 223, 2014.

[10] D. B. Brito, L. M. Araújo, A. G. d'Assunção and R. H. C. Maniçoba, "A Minkowski fractal Frequency Selective Surface with high angular stability", In Proceedings of 2013 SBMO/IEEE MTT-S International Microwave and Optoelectronics Conference (IMOC), 1 - 4, 2013. 